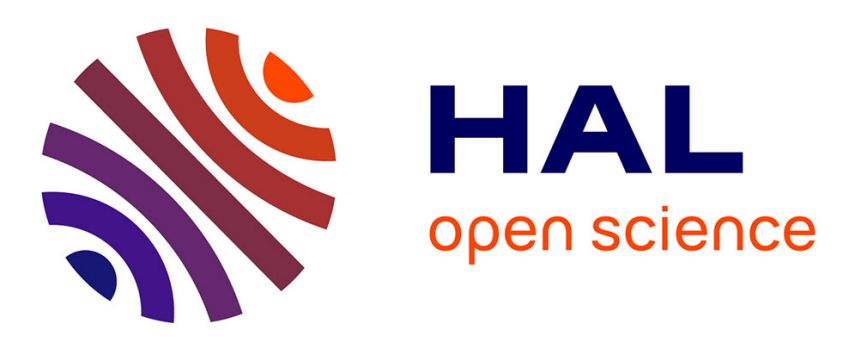

\title{
Modeling Embryonic Cleavage Patterns
}

Dmitry Ershov, Nicolas Minc

\section{- To cite this version:}

Dmitry Ershov, Nicolas Minc. Modeling Embryonic Cleavage Patterns. Methods in Molecular Biology, 1920, pp.393-406, 2019, 10.1007/978-1-4939-9009-2_24. hal-02381740

\section{HAL Id: hal-02381740 https://hal.science/hal-02381740}

Submitted on 26 Nov 2019

HAL is a multi-disciplinary open access archive for the deposit and dissemination of scientific research documents, whether they are published or not. The documents may come from teaching and research institutions in France or abroad, or from public or private research centers.
L'archive ouverte pluridisciplinaire HAL, est destinée au dépôt et à la diffusion de documents scientifiques de niveau recherche, publiés ou non, émanant des établissements d'enseignement et de recherche français ou étrangers, des laboratoires publics ou privés. 
Metadata of the chapter that will be visualized online

\begin{tabular}{|c|c|c|}
\hline Chapter Title & \multicolumn{2}{|c|}{ Modeling Embryonic Cleavage Patterns } \\
\hline Copyright Year & \multicolumn{2}{|l|}{2019} \\
\hline Copyright Holder & \multicolumn{2}{|c|}{ Springer Science+Business Media, LLC, part of Springer Nature } \\
\hline \multirow[t]{8}{*}{ Author } & Family Name & Ershov \\
\hline & Particle & \\
\hline & Given Name & Dmitry \\
\hline & Suffix & \\
\hline & Organization & Institut Jacques Monod, CNRS UMR7592 \\
\hline & Address & Paris, France \\
\hline & Organization & Université Paris Diderot \\
\hline & Address & Paris, France \\
\hline \multirow[t]{9}{*}{ Corresponding Author } & Family Name & Minc \\
\hline & Particle & \\
\hline & Given Name & Nicolas \\
\hline & Suffix & \\
\hline & Organization & Institut Jacques Monod, CNRS UMR7592 \\
\hline & Address & Paris, France \\
\hline & Organization & Université Paris Diderot \\
\hline & Address & Paris, France \\
\hline & Email & nicolas.minc@ijm.fr \\
\hline Abstract & \multicolumn{2}{|c|}{$\begin{array}{l}\text { The division patterns of early invertebrate and vertebrate embryos are key } \\
\text { to the specification of cell fates and embryo body axes. We here describe a } \\
\text { generic computational modeling method to quantitatively test } \\
\text { mechanisms which specify successive division position and orientation } \\
\text { of eggs and early blastomeres in 3D. This approach should serve to } \\
\text { motivate and guide future experimental work on the mechanisms } \\
\text { controlling early embryo morphogenesis. }\end{array}$} \\
\hline $\begin{array}{l}\text { Keywords } \\
\text { (separated by '-') }\end{array}$ & \multicolumn{2}{|c|}{$\begin{array}{l}\text { Early embryos - Division positioning - Microtubules - Asters - Cell shape - } \\
\text { Maternal domain - Yolk }\end{array}$} \\
\hline
\end{tabular}




\title{
Author's Proof
}

\section{Chapter 24}

\section{Modeling Embryonic Cleavage Patterns}

Dmitry Ershov and Nicolas Minc

\begin{abstract}
The division patterns of early invertebrate and vertebrate embryos are key to the specification of cell fates 5 and embryo body axes. We here describe a generic computational modeling method to quantitatively test 6 mechanisms which specify successive division position and orientation of eggs and early blastomeres in 3D. 7 This approach should serve to motivate and guide future experimental work on the mechanisms controlling 8 early embryo morphogenesis.

Key words Early embryos, Division positioning, Microtubules, Asters, Cell shape, Maternal domain, 10 Yolk

\section{Introduction}

Early embryo development of both invertebrate and vertebrate 13 species begins with the cleavage period. During cleavage, the egg 14 undergoes a stereotyped choreography of subsequent reductive 15 divisions that transform the one-cell egg into a multicellular 16 embryo [1]. Cleavage patterns are not random, but usually follow 17 a well-ordered set of specific position and orientation of blastomere 18 divisions. Those patterns are most often conserved among large 19 groups of species, such as in amphibians, fishes, and echinoderms, 20 and serve as prime examples of developmental robustness. Mechan- 21 isms which control cleavage geometries remain however poorly 22 understood. This is in part because this period is driven by maternal 23 material in the embryo, and thus poorly amenable to genetic 24 screens or manipulations [2]. In addition thorough live imaging 25 of large eggs and blastomeres implicates sophisticated methodolo- 26 gies [3], or may even be impaired given the opacity of many 27 embryos. Factors that have been directly and indirectly implicated 28 in the regulation of division plane positioning during cleavage 29 include cell shapes [4-7], yolk layers [8,9], and maternal cortical 30 polarity domains [10-13]. Those may affect the organization or 31 dynamic properties of astral microtubules which exert forces and 32 


\section{Author's Proof}

Dmitry Ershov and Nicolas Minc

torques to position and orient asters and mitotic spindles that 33 specify division planes.

Recent experimental work in echinoderms, fish, and frog 35 embryos has suggested that division plane specification in large 36 eggs and blastomeres is instructed from interphase/anaphase 37 microtubule (MT) asters which fill the large cellular volume, and 38 probe cell shape through length-dependent MT forces $[6,7]$. This 39 mechanism aligns the division axis with the cell shape long axis, and 40 has been proposed to serve as a default cue guiding division plane 41 orientation and position in early embryos [14, 15]. Given the 42 presence of asymmetric divisions in some blastomeres of many embryos, cell shape effect may be overridden or biased by additional cues such as vegetal polarity domains in echinoderms or by yolk layers in fish and amphibians, which alter the distribution of MT forces around cells.

We have recently implemented 3D computational models to predict division position/orientation as a function of various inputs [16]. Those models compute in a systematic manner the forces and torques exerted by astral MTs on centrosome pairs at the center of asters and output a mechanical equilibrium which corresponds to the preferred division position and orientation. Those models are highly valuable to dissect the basics of cleavage patterns in many embryos. In here, we report on a set of scripts and methodologies to implement this modeling approach to test the contribution of various cues to the division patterns of early embryos.

\section{Materials}

Computer equipped with Mathworks Matlab (Version 2015a and above) and Surface Evolver [17]: There is no special requirement for computers, but it is recommended for better performance to have a fast CPU. On a good system a simulation of 10,000 iterations with calculation of a torque map (see here after) typically takes 5-10 min.

Programs may be downloaded from:

Division Prediction Program (DPP):

http://www.minclab.fr/wp-content/uploads/2018/01/ 67

DPP-Package.zip

Surface Evolver

http://facstaff.susqu.edu/brakke/evolver/evolver.html

3.1 Generating 3D Cell Shapes
The initial input for the program is the 3D shape of a cell or a group 72 of cells in an embryo, for instance, defined by the 3D coordinates of 73 cell surfaces. This serves as a spatial reference to run a prediction on 


\section{Author's Proof}

A

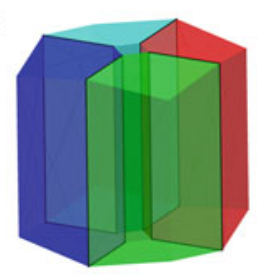

Raw surfaces
B

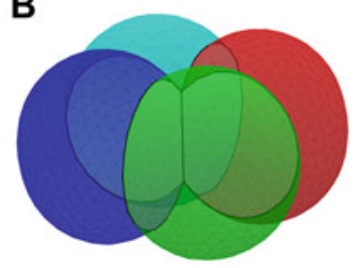

Surfaces of minimal energy

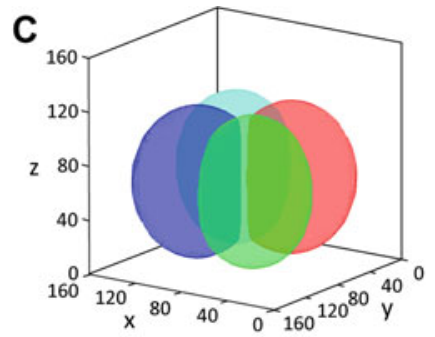

Surfaces imported to DPP

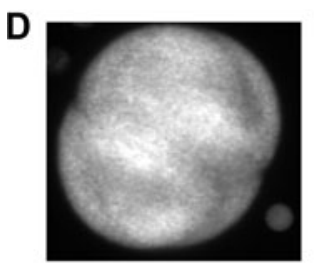

Experimental image stack

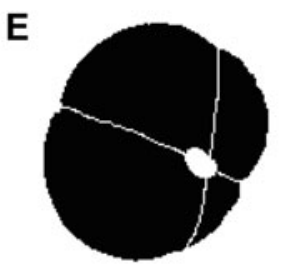

Segmented image stack
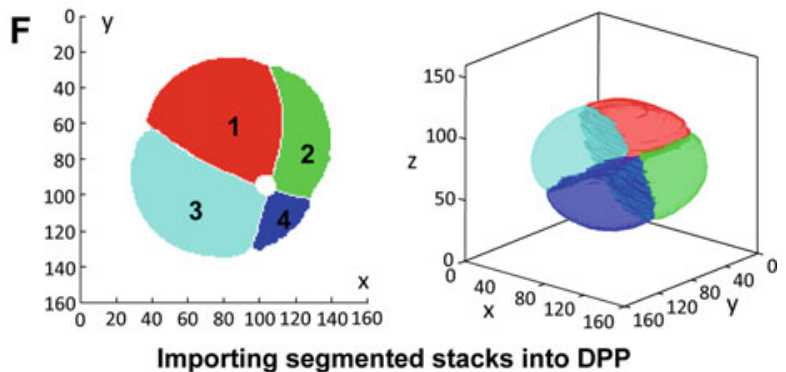

Fig. 1 Creating 3D Cell Shape inputs. (a) Raw surfaces for a four-cell embryo in Surface Evolver (b)equilibrated shape with surfaces of minimal energy obtained in SE. (c) Equilibrated 3D shape imported to DPP with Matlab. (d) Experimental image projected stack of a four-cell sea urchin embryo. (e) Segmented image stack. (f) Interface to import 3D segmented stack in Matlab for DPP, through manual connection of 2D segments in 3D

the position/orientation of the site of cell division. 3D shapes may 75 be artificially generated using the surface minimization software 76 Surface Evolver [17], or from a 3D segmented stack from labeled 77 embryos imaged with a confocal or a two-photon microscope 78 (Fig. 1).

3.1.1 Generating an Input 3D Shape with Surface Evolver
We here provide some initial guidelines to generate representative 80 3D shapes that closely resemble real physical egg and blastomere 81 shapes with the software Surface Evolver (SE). This software itera- 82 tively finds the surface of minimal energy under given constraints 83 (Fig. la, b). Constraints include final volume, surface tension 84 values, and confinement. The language of SE is rich and for details 85 on structures, constraints, macros, or function definition we refer 86 the readers to its web page.

SE requires input files that contain information on the topol- 88 ogy and constraints of the studied surfaces. The topology is 89 described by a simple mesh (Fig. la), elements of which are 90 named and organized according to the rules of SE. The mesh 91 describing topology is defined by vertices and their coordinates, 92 oriented edges linking two vertices, oriented faces consisting of 93 three edges, and bodies consisting of $\mathrm{N}$ faces. The constraints 94 describe the behavior of the surface; for example coordinates can 95 


\section{Author's Proof}

Creating Shapes with Surface Evolver be restricted to an arbitrary space (like a cylinder) or additional energy may be assigned to arbitrary faces, to mimic effects of differential surface tension around adhering blastomeres for instance [16]. We provide several input files in the folder DPP package $\backslash D P P$ files\generating_shapes_from_SE\generic shapes\, which can be used to create one-cell spheres, rods, and typical two-cell, four-cell, or eight-cell stage embryos. In the DPP package, we further provide more detailed guidelines to create other shapes and constraints with SE. For the method description below we will use the example file " 4 cells - confined in sphere - raw.fe".

1. Open Matlab. Navigate to the DPP Package folder in Matlab, right-click on it, and click "add to path/Select folders and subfolders". Navigate to the folder "DPP package $\backslash D P P$ program〉" where three scripts named "launcher" are placed; you will work from here.

2. Open the script "launcher_l_generate_shapes.m" with 111 Matlab. Set the path where you will store the shapes, simulation parameters, and results. For instance, if you want to store your cells and their simulations in a folder "C:|Data|my cell" you set the variable root_path $=$ "C: $\mid$ Data $\mid$ my cell".

3. Run the function that generates the folders shape_- 116 paths = fl_generate_paths(root_path) by selecting the two 117 abovementioned lines and pressing F9 to evaluate/run. This 118 will create the folder "C:|Data|my cell" and inside it a folder 119 "C:|Data $\mid$ my cell $\backslash$ shape_source". In the latter you will keep all 120 cell shape-related files.

4. Copy the file " 4 cells - confined in sphere - raw.fe" into the 122 folder "C:|Data $\mid$ my cell $\backslash$ shape_source" and double-click the 123 input file to open it in SE.

5. To see the surface, press "s" to enter the graphic mode 125 (Fig. la). Then "q" to quit the graphic mode and return to 126 $\mathrm{SE}$ command terminal. You may zoom in and out (press Z 127 when graphic window is active) or rotate (press $\mathrm{R}$ when graphic 128 window is active) the surface using the mouse.

6. To iteratively find minimal energy configuration (Fig. 1b), the user needs to perform subsequent refinements of the mesh and calculation of novel surface coordinates. Type " $r$ " to refine the initial mesh (subdivide); this will increase the number of vertices. Then "g10" to make ten iterations of surface minimization (g100 will make 100, etc.). Refine the mesh again with " $\mathrm{r}$ ". Refine and reiterate calculations several times until you find that the shape is stable and does not change significantly with the following iterations. As a start for division prediction, we recommend to refine the mesh to reach a number of typically 1000-2000 vertices.

19 


\section{Author's Proof}

Translating the SE Surface into a Matlab Matrix with Labeled Bodies
3.1.2 Generating an Input 3D Shape from an Imaging Stack of a Real Embryo
7. Save the final file by typing " $d$ " to save the data in one file in SE 141 format. Use a different name than the original file, e.g., 142 "4_cell_final".

8. Type "export_lists( $)$ " in the SE terminal to extract the data in 144 separate lists for vertices, edges, faces, and bodies. Note that 145 this function has been added to the example files to facilitate 146 the following steps, but is not a default function of SE. In the 147 package we provide this function which can be copied into any 148 SE function. At the end of this process, you should end up with 149 two SE files (.fe), one for the initial shape, and one for the final, 150 and several .txt files for edges, faces, vertices, and bodies. 151

1. In Matlab open the script "launcher_1_generate_shapes.m". 153 Find the function "f2_extract_bodies_from_SE(shape_paths, 154 $[\mathrm{X}, \mathrm{Y}])$ " and run it by selecting the line and pressing F9. The 155 numbers in the brackets are the size in pixels of a Matlab matrix 156 that will comprise the SE surface; for instance: $[X, Y]=[200,157$ 240 ] pixels. Any size in any proportion can be used, but large 158 matrices may take much longer to simulate. Typically, we use 159 matrices of the sizes ranging from $150 \times 150$ to $200 \times 200160$ pixels; $\mathrm{X}$ and $\mathrm{Y}$ may be set unequal ( $\mathrm{Z}$ is automatically recalcu- 161 lated from shape's dimensions and given X and Y to ensure the 162 best fit of the shape within the matrix).

2. Matlab will ask you to provide the name of the SE file from 164 which you exported the lists (its name was used as a part of 165 those lists and will be used to find them); in our case it is " $\mathrm{C}$ : 166 Data|my cell $\backslash$ shape_source $\backslash 4$ cells - confined in sphere - raw. 167 fe". The program will read the raw coordinates in ".txt" files 168 and save them as "C:|Data $\mid$ my cell $\mid$ shape_source $\mid$ shape_data. 169 mat", after which it will translate them to matrix coordinates, 170 label them according to the body lists, and save those as " $\mathrm{C}: 171$ \Data|my cell|shape_source|labeled_bodies.mat" in the same 172 folder.

3. In Matlab open the script "launcher_l_generate_shapes.m". 174 Find the function "f3_show_labeled_bodies(shape_paths)" 175 and run it. It will find the file "labeled_bodies.mat" and show 176 the labeled bodies (Fig. Ic). If everything went fine up to this 177 point, the system is ready to run division predictions (Subhead- 178 ing 3.2).

In many instances, one may need to input the direct 3D shape from 181 a cell or a group of cell in an embryo/tissue, and compare experi- 182 mental division orientation with model predictions. Those shapes 183 may be experimentally obtained from a Z-stack taken on a labeled 184 embryo with confocal, light-sheet, or two-photon microscopy 185 (Fig. 1d). Here we solely provide a method to convert a 3D 186 


\section{Author's Proof}

segmented stack into an input file to run the division prediction 187 program. Segmentation of 3D shapes may be achieved with ImageJ or Imaris, and will largely differ depending on the embryo, labels, and microscopes. For our purpose, input segmented stacks must be black inside and white outside. The voxel should be cubic (it should span equally in all dimensions: $\mathrm{V}_{\mathrm{x}}=\mathrm{V}_{\mathrm{y}}=\mathrm{V}_{\mathrm{z}}$ ) and the final segmentation is recommended to span not less than $100 \times 100 \times 100$ pixels (Fig. le).

In the folder "DPP package|DPP files\generating_shapes_from_experiment|" we provide an example stack of a segmented fourcell sea urchin embryo called "4CellUrchin_final.tif" which we use to present this part of the method.

1. Open Matlab. Navigate to the DPP Package folder in Matlab, right-click on it, and click "add to path/ Select folders and subfolders". Navigate to the folder "DPP package $\backslash D P P$ programl" where three scripts named "launcher" are placed; you will work from here.

2. Open the script "launcher_1_generate_shapes.m" with Matlab. Set the path where you will store the shapes, generated cells, and their simulations. For instance, if you want to store your cells and their simulations in a folder "C:|Data|my cell" you set the variable: root_path $=$ "C:|Data $\backslash$ my cell". Run the function that generates the folders shape_paths $=\mathrm{fl} \_$generate_paths(root_path) by selecting the two abovementioned lines and pressing F9 to evaluate/run. This will create the folder "C: $\backslash$ Data $\backslash$ my cell" and inside it a folder "C:|Data|my cell|shape_source". Copy the file "4CellUrchin_final.tif" into this folder.

3. In Matlab open the script "launcher_l_generate_shapes.m", find the function "f2_extract_bodies_from_IJ", and run it by selecting the line and pressing F9. It will offer to choose a segmentation stack; choose "4CellUrchin_final.tif".

4. You will be taken to an interface allowing you to manually connect $2 \mathrm{D}$ regions in the stack to reconstitute the proper 3D shape of your embryo/tissue (Fig. If). For this, you will need to manually label $2 \mathrm{D}$ regions belonging to one cell from slice to slice (Fig. If, left). You can scroll through the whole stack using the mouse wheel. Then set a label number $\mathrm{N}$, by pressing a numeric key on the keyboard, and label a 2D region with this $\mathrm{N}$ number by clicking on each $\mathrm{z}$-slice as it appears. The program takes the user to the next slice automatically after the click. After all slices of one cell have been connected, you can visualize the result in $3 \mathrm{D}$ by pressing $\langle\mathrm{v}\rangle$ (Fig. If, right). After all cells have been reconstructed, it is recommended to clean the stack from lone pixels by pressing $\langle\mathrm{c}>$ and visualize in $3 \mathrm{D}$ again; the final result should look similar to Fig. If. Export the resulting bodies by pressing $<\mathrm{s}>$. This will create 


\section{Author's Proof}

\subsection{Predicting Division Position and Orientation in $3 D$}

3.2.1 Setting a Choice of Parameters for the Simulations a Matlab file “C:|Data $\backslash$ my cell $\mid$ shape_source|labeled_bodies. 233 mat". If everything went fine up to this point, the system is 234 ready to run division predictions (Subheading 3.2). 235

In this part we describe the methods to use the scripts which allow 237 to make a prediction on the orientation and position of the division 238 axis. This prediction will have as inputs 3D cell shapes defined 239 following the preceding paragraphs, and parameters, which relate 240 to the distribution of astral microtubule (MT) forces within cells 241 and to simulation procedures. As such, it is important to state that 242 those models can solely be useful to study cell division in cell types 243 and systems in which MTs are prime contributor of nuclei/spindle 244 position, as in most vertebrate and invertebrate early embryos 245 $[15,14]$. In our model, MT forces may depend on the spatial 246 distribution of MTs around centrosomes, the length of MTs, or 247 the presence of cortical domains. MT lengths are defined by cell 248 shape and/or presence of MT excluding structures in cells, such as 249 neighboring asters or yolk. 250

1. In Matlab open the script "launcher_2_generate_cell.m", 252 which contains a set of parameters for the cell simulation. If 253 you run this program following immediately Subheadings 254 "Creating Shapes with Surface Evolver" or "Translating the 255 SE Surface into a Matlab Matrix with Labeled Bodies", the 256 path to your cell is already set. Else, redo Subheading "Creating 257 Shapes with Surface Evolver", step 2 or Subheading "Translat- 258 ing the SE Surface into a Matlab Matrix with Labeled Bodies”. 259

2. Define cell to simulate: "body_ind =". If you have several cells (like in the four-cell embryo mentioned above), this is the index of the particular cell that will be used as the shape input. In the part described in Subheading 3.1.2, step 4, to generate shapes from real $3 \mathrm{D}$ images, this number is the one defined by the user during the $3 \mathrm{D}$ reconstruction.

3. Define microtubule pulling force parameters. As described in [16], the general formula used to define the force exerted by each MT in the asters is

$$
\begin{aligned}
F= & L^{\wedge} \text { Betal }+ \text { pullfactor }{ }^{*} L^{\wedge} \text { Beta } 2 \\
& + \text { pullfactor_exp }{ }^{*} 2(L / \text { Unit_branch })
\end{aligned}
$$

The first term corresponds to pulling in the cytoplasm in pro- 269 portion to MT length, with an exponent factor, "Betal", 270 reflecting putative nonlinearities, which by default is set to 271 3 [6]. The second and third terms reflect pulling from a polar 272 cortical domain (whose location and size are defined hereafter). 273 "Beta2" reflects an exponent for cortical pulling, and "pull- 274 factor" the strength of this effect. Beta2 is set by default to 2 to 275 


\section{Author's Proof}

reflect a situation in which diluted motors are limiting pulling from the domain [18]. The last term reflects a situation in which MT number at the domain is limiting pulling, and possesses an exponential dependence term, defined through the parameter "Unit_branch", to mimic the presence of branching in large embryonic asters (a situation likely irrelevant for somatic cells). This parameter has a default value of 6.75 , as experimentally measured in sea urchins [6]. The strength of this term is defined by the parameter "pullfactor_exp". If both parameters "pullfactor" and "pullfactor_exp" are set to 0, then the script will not propose you to position and size a cortical domain. In such case, you may directly proceed to Subheading 3.2.1, step 7 .

4. Define exclusion regions for microtubule growth. Microtubule effective length can be corrected for the presence of intracellular structure such as yolk which affects MT growth in a dosedependent manner. This is done by correcting the angular density of MTs in aster by a factor $1-[$ yolk $] / \eta$, with [yolk] the local yolk concentration and $\eta$ a sensitivity parameter for how MTs are affected by the yolk. This implies defining the location of the yolk gradient, its orientation, and its sharpness, as detailed in [16]. The parameter "gradyolk" defines the sharpness of the yolk gradient (if set to 0, MTs are uniform inside the cell shape). "erfoffset" is the offset of the sharpest gradient point in pixels. "direction_grad" is used for the direction of the yolk gradient, and is defined by polar $(\theta)$ and azimuthal $(\varphi)$ angles, in degrees, counted in a standard convention: $\theta_{y}$ is counted from the $z$-axis; $\varphi_{y}$ is counted counterclockwise from the $x$-axis (Fig. 2b). "MTstab" defines the sensitivity of MTs to yolk, $\eta$, and is set to 1 by default. Importantly, the shape of the yolk gradient will appear in the graphic interface upon running the script, and can thus be adjusted back (Fig. $2 \mathrm{~d}$ and Subheading 3.2.1, step 8).

5. Aster parameters: Parameters related to MT asters include initial position and orientation of asters from where to start a simulation, extension angle of asters, and distance between centrosomes. "anglelimit_deg" reflects the extension of asters in degrees ( $\beta$ in Fig. 2a). "theta" is the starting polar angle in spherical coordinates, counted from the $z$-axis ( $\theta$ in Fig. $2 a)$. "phi" is the starting azimuthal angle in spherical coordinates; counted counterclockwise from the $x$-axis ( $\varphi$ in Fig. 2a). "startingxyz" is the initial position of nuclei/spindle center in pixels, defined by three Cartesian coordinates $[\mathrm{x}, \mathrm{y}, \mathrm{z}]$. If empty, the initial position will be at the calculated "centroid" of the cell shape. "Nucsize" is the distance between centrosomes in pixels (spindle or nuclear size). "Nucexcl" is a parameter used to calculate an exclusion volume ensuring that the nucleus/ 


\section{Author's Proof}

A

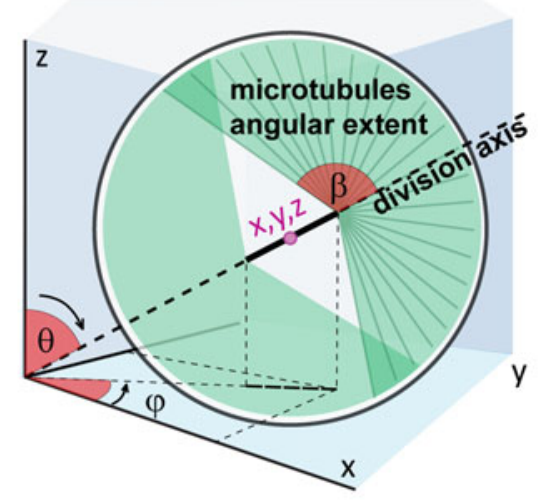

Aster parameters

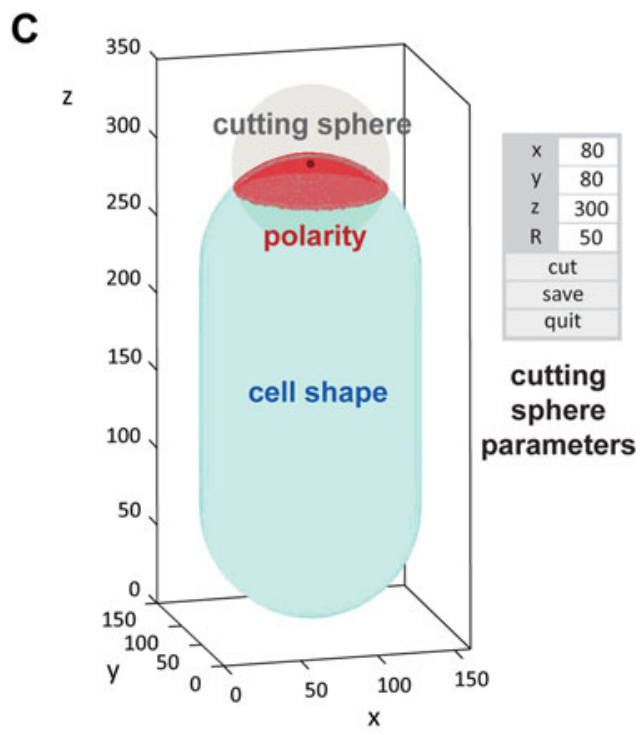

Defining polarity domain in DPP
B

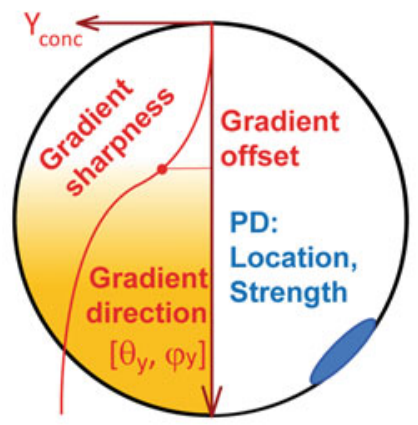

Yolk gradient $(\mathrm{Y}) \quad$ Polarity domain (PD)

Pulling force effectors

D

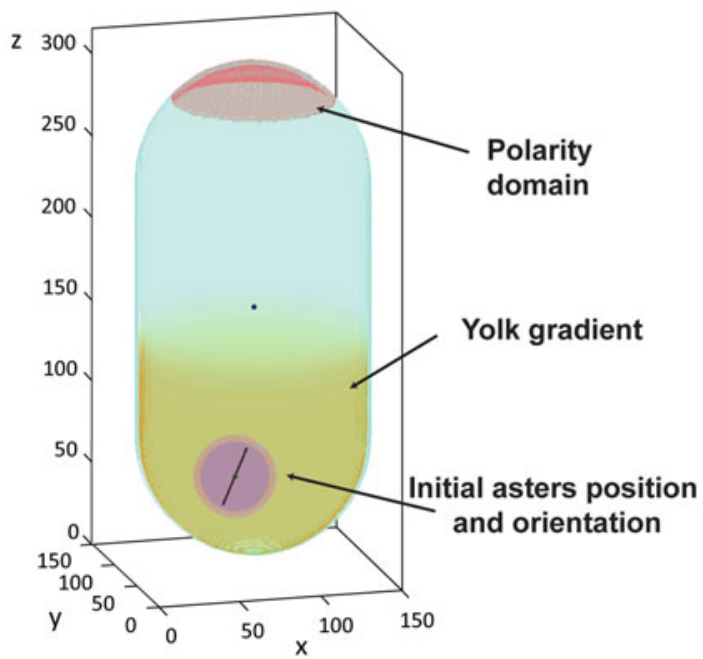

Polarity domain and Yolk gradient in a generated cell in DPP

Fig. 2 Input parameters for predicting division position and orientation. (a) Microtubule aster parameters. Polar and azimuthal angles $(\theta$ and $\varphi)$ defining the division axis angle; and position of the center $0(\mathrm{x}, \mathrm{y}, \mathrm{z})$; angular extent of microtubules ( $\beta$, counted from the division axis). (b) Parameters affecting microtubule pulling forces. MT growth may be tuned by the presence of yolk gradients $(Y)$, with the concentration gradient defined by a direction $\left(\theta_{y}, \varphi_{y}\right)$, an offset from the shape centroid (along the gradient direction), and sharpness. A polarity domain (PD) with a given size, location, and MT pulling strength may also be added as an input for simulations. (c) Matlab interface to create a polarity domain: a cutting sphere of defined center and radius intersects the 3D cell shape to define the domain. (d) Visualization of the cell with its major simulation parameters used as initial inputs to predict division position and orientation 


\section{Author's Proof}

3.2.2 Starting a Simulation spindle stays inside the cell during the simulation. Nucexcl cannot be $<1$; typical values are 1.2-1.4.

6. After all the parameters have been set, run the whole script by pressing the "Run" button in the Matlab Editor panel. This will create the parameter structures and feed them to the class "DPP_cell" needed for the simulation.

7. If you have set polarity parameters different than 0 (see Subheading 3.2.1, step 3), you will be offered to position and size a polarity domain. For this you will be brought directly to a graphic interface (Fig. 2c). The domain is defined as the intersection of the $3 \mathrm{D}$ cell shape with a cutting sphere. Use the interface to define values for the coordinates $(x, y, z)$ of the sphere center and radius $(R)$, and press "Cut" to visualize the domain. Once set, press the button "Save" to set the domain for the simulation. If you press "Quit" this will set the pulling pre-factors to 0 and run a simulation purely based on cytoplasmic MT forces and cell shape.

8. If everything was set correctly, the cell with polar domain, yolk gradient, and initial orientation/position of nuclei/spindle will be shown as in Fig. 2d. Corresponding parameters will be automatically saved in a new folder "cell_n". Each time you run this script the cell folder index " $n$ " increases automatically, e.g., "C:|Data $\mid$ my cell|cell_l” and "C:|Data $\mid$ my cell $\mid$ cell_2".

The principle of the simulation is based on a random walk around 347 the 3D position and orientation of the nucleus/spindle by three center coordinates $(\mathrm{x}, \mathrm{y}, \mathrm{z})$ and two angles $(\theta$ and $\varphi)$. At each iteration one of those variables is changed, the force and torques of all MTs on the nucleus/spindle are calculated and compared to the previous step in order to converge toward an equilibrium of minimal forces and torques [16]. The simulation thus comes with parameters to optimize time, precision, or accuracy, which need to be defined by the user.

1. Following the preceding section, open the script "launcher_3_generate_simulation.m".

2. Set visualization parameters.

With "show_log $=1$ " the program outputs to the Matlab 359 command line information on what parameter has been varied and how forces are calculated. If not required, set to 0 .

With "show_visually $=\mathrm{N}$ " (higher than 0$)$ the program will 361 display the nucleus position and orientation of each $\mathrm{N}$ iterations. If set to 0 , there will be no visualization, which may improve simulation speed. 
“auto_save_nucleus_run $=1$ ": the program will save the run of 367 aster centers (trajectory during simulation) as a PNG picture. 368 "auto_save_torque_map $=1$ ": the program will save a torque 369 map as a PNG (see below). "auto_save_state_history =1": the 370 program will save the history of varying parameters during the 371 simulation as a PNG.

"auto_save_split_image $=1$ ": the program will save an image 373 of a divided cell (with two daughter cells) as a PNG. 374

4. Random walk parameters.

“angle_btw_mt_deg” defines the angle (in degrees) between 376 microtubules (used for both MT polar and azimuthal angle), 377 and somehow reflects the precision of the simulation. If set to 378 10, for instance, the forces will be calculated along MTs in the 379 aster spanning each $10^{\circ}$ in both polar and azimuthal angles. 380 The larger the angle between MTs, the faster the calculations 381 are, but the less precise also. It is recommended to use large 382 steps (10-20) to quickly and roughly find equilibrium position 383 and then use lower angles (1-3) to refine this position and find 384 proper orientation (see below). 385 “jump_chance $=$ ": Probability of jumping to a new configura- 386 tion, even if it has been estimated to be less favorable (forces 387 and torques are higher) than the previous state. If set to 388 10 , then the chance to make a jump is $10 \%$. 389 "Inertia $=":$ It is the probability to vary the same parameter in 390 the same manner if its previous variation led to a more favorable 391 state (100: always use previous variation; 0: randomly pick a 392 new variation).

"duration_equilibrium =": The number of iterations required 394 for a new state to be tested for equilibrium. If x, y, and $\mathrm{z}$ do not 395 change for this number of iterations, a position is considered 396 equilibrated.

"duration_full =": It sets the maximum number of iterations 398 (use typically $>5000$ ).

5. The torque map is important as it informs the stability of the 400 current division orientation as compared to others. For 401 instance in shapes which are roughly isotropic, this map 402 would be flat with no sharp torque minima [16]. The torque 403 map is a $2 \mathrm{D}$ matrix of absolute values of torques at each 404 orientation angle (Fig. 3d), calculated as: $\quad 405$ $T\left(\varphi_{i}, \theta_{j}\right)=\sqrt{T_{\varphi_{i}}^{2}+T_{\theta_{j}}^{2}} \quad 406$ Torque map parameters are as follows: $\quad 407$ “torque_map_angle_step =": This sets a precision step for 408 torque map calculation. If set to 10 , torque map calculation 409 will sample division orientations each $10^{\circ}$ for both $\theta$ and $\varphi . \quad 410$ “auto_calc_torque_map $=1$ ": The program will calculate a 411 torque map automatically after the simulation (it may take a 412 


\section{Author's Proof}

Dmitry Ershov and Nicolas Minc

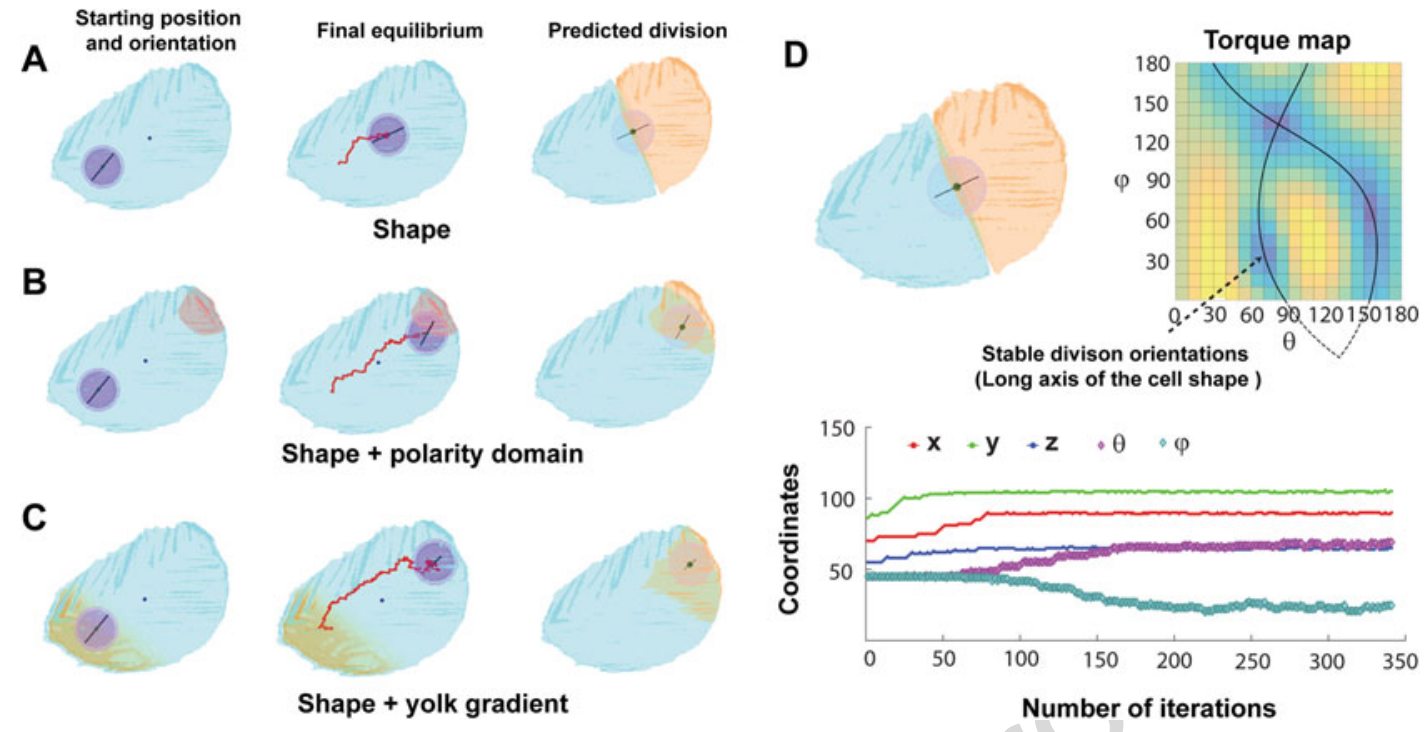

Fig. 3 Example of DPP simulation results. (a) (Left) Cell shape corresponding to one blastomere of the four-cell embryo from Fig. 1d is used as the sole input to predict division position and orientation. (Middle) After simulation, asters are positioned at the cell center and oriented along the long axis of the 3D shape. (Right) The predicted divided cell is then generated. (b) Same as in (a) but with the presence of a polarity domain pulling on asters. Note the strong asymmetry in cell division. (c) Same as in (a) but with a yolk gradient which creates a shift in the final position of asters away from yolk layers. (d) Simulation as in (a) with corresponding torque map, computing the torque exerted on asters as a function of all possible 3D angles. Torque minima correspond to stable orientations, highlighted by the black lines. (Bottom) Evolution of angular and position coordinates during the random walk, as a function of iterative step number of the walk

long time depending on the precision of the map). If not required, set to 0 .

6. Once parameters are set, start the simulation by selecting all the lines from "current_simulation" down to "current_simulation. run" and pressing F9.

7. The simulation will end either if it ran out of iterations (from the maximum number allowed) or if it found an equilibrium which did not move during a number of iteration set by the parameter inertia. The final state of the simulation will be saved automatically in the cell folder, i.e., "C: $\mid$ Data $\mid$ my cell $\mid$ cell_n $\mid$ " with the prefix corresponding to the index of current instance: "run_1", "run_2", and so on. Windows showing the results of the simulation will open, depending on the autosave flags (see Fig. 3 for examples of simulation output).

8. If you want to refine the simulation, with more MTs to improve precision, or with a more detailed torque map, use the section "Refine division orientation", insert new numbers, and run by selecting lines from "current_simulation.reset" down to "current_simulation.show_division_plane”. 


\section{Author's Proof}

9. Finally, one can use old cell shape and parameters to rerun 432 simulation. To that aim, open the folder with the cell of inter- 433 est, e.g., "C: $\backslash$ Data $\backslash$ my Cell|cell_l", and drag and drop the file 434 "cell.mat" into Matlab workspace to load it. In the script 435 "launcher_2_generate_cell.m" run the line "current_cell. 436 show", and visualize the initial state of the cell visually. You 437 can also inspect all the properties of the cell by typing "curren- 438 t_cell” in the command line and pressing enter. Then restart 439 from Subheading 3.2.2, step 1 to run a new simulation. $\quad 440$

\section{References}

1. Gilbert S (2010) Developmental biology, 9th edn. Developmental Biology Sinauer Associates, Sunderland (MA)

2. Pelegri F, Dekens MP, Schulte-Merker S, Maischein HM, Weiler C, Nusslein-Volhard C (2004) Identification of recessive maternaleffect mutations in the zebrafish using a gynogenesis-based method. Dev Dyn 231 (2):324-335. https://doi.org/10.1002/dvdy. 20145

3. Olivier N, Luengo-Oroz MA, Duloquin L, Faure E, Savy T, Veilleux I, Solinas X, Debarre D, Bourgine P, Santos A, Peyrieras N, Beaurepaire E (2010) Cell lineage reconstruction of early zebrafish embryos using label-free nonlinear microscopy. Science 329 (5994):967-971

4. Hertwig O (1884) Das Problem der Befruchtung une der Isotropie des Eies, eine Theory der Vererbung. Jenaische Zeitschrist

5. Hertwig O (1893) Ueber den Werth der ersten Furchungszellen fuer die Organbildung des Embryo. Experimentelle Studien am Froschund Tritonei. Arch mikr Anat xlii:662-807

6. Minc N, Burgess D, Chang F (2011) Influence of cell geometry on division-plane positioning. Cell 144(3):414-426
7. Wuhr M, Tan ES, Parker SK, Detrich HW, 3rd, Mitchison TJ (2010) A model for cleavage plane determination in early amphibian and fish embryos. Curr Biol 20 (22):2040-2045. doi:https://doi.org/10.1016/j.cub.2010.10. 024

8. Neff AW, Wakahara M, Jurand A, Malacinski GM (1984) Experimental analyses of cytoplasmic rearrangements which follow fertilization and accompany symmetrization of inverted Xenopus eggs. J Embryol Exp Morphol 80:197-224

9. Yokota H, Neff AW, Malacinski GM (1992) Altering the position of the first horizontal cleavage furrow of the amphibian (Xenopus) egg reduces embryonic survival. Int $\mathrm{J}$ Dev Biol 36(4):527-535

10. Sardet C, Paix A, Prodon F, Dru P, Chenevert J (2007) From oocyte to 16-cell stage: cytoplasmic and cortical reorganizations that pattern the ascidian embryo. Dev Dyn 236 (7):1716-1731. https://doi.org/10.1002/ dvdy.21136

11. Dan K (1979) Studies on unequal cleavage in sea urchins I. Migration of the nuclei to the vegetal pole. Develop Growth Differ 21 (6):527-535 


\section{Author's Proof}

12. Leonard JD, Ettensohn CA (2007) Analysis of dishevelled localization and function in the early sea urchin embryo. Dev Biol 306 (1):50-65

13. Gonczy P (2008) Mechanisms of asymmetric cell division: flies and worms pave the way. Nat Rev Mol Cell Biol 9(5):355-366. https://doi. org $/ 10.1038 / \mathrm{nrm} 2388$

14. Mitchison T, Wuhr M, Nguyen P, Ishihara K, Groen A, Field CM (2012) Growth, interaction, and positioning of microtubule asters in extremely large vertebrate embryo cells. Cytoskeleton 69(10):738-750. https://doi.org/ $10.1002 / \mathrm{cm} .21050$
15. Hasley A, Chavez S, Danilchik M, Wuhr M, Pelegri F (2017) Vertebrate embryonic cleavage pattern determination. Adv Exp Med Biol 953:117-171. https://doi.org/10.1007/ 978-3-319-46095-6_4

16. Pierre A, Sallé J, Wühr M, Minc N (2016) Generic theoretic models to predict division patterns of cleaving embryos. Dev Cell 39:1-16

17. Brakke KA (1992) The surface evolver. Exp Math 1(2):141-165

18. Grill SW, Hyman AA (2005) Spindle positioning by cortical pulling forces. Dev Cell 8 (4):461-465 


\section{Author Queries}

Chapter No.: 24

370791_2_En

\begin{tabular}{|l|l|l|}
\hline Query Refs. & Details Required & Author's response \\
\hline AU1 & $\begin{array}{l}\text { Please check and confirm if the affiliations are } \\
\text { presented correctly. }\end{array}$ & \\
\hline AU2 & $\begin{array}{l}\text { Please check the hierarchy of the section head- } \\
\text { ings and confirm if correct. }\end{array}$ & \\
\hline
\end{tabular}

\section{Author's Proof}

